\title{
Clinical efficacy of the classic laryngeal mask airway in elderly patients: a comparison with young adult patients
}

\author{
Eun Mi Kim ${ }^{1}$, Min-Soo Kim²,3 Bon-Nyeo Koo ${ }^{2,3}$, Jeong-Rim Lee ${ }^{2,3}$, \\ Young Sub Lee ${ }^{1}$, and Jae Hoon Lee ${ }^{2,3}$ \\ ${ }^{I}$ Department of Anesthesiology and Pain Medicine, Kangnam Sacred Heart Hospital, Hallym University College of \\ Medicine, ${ }^{2}$ Department of Anesthesiology and Pain Medicine, Severance Hospital, ${ }^{3}$ Anesthesia and Pain Research \\ Institute, Yonsei University College of Medicine, Seoul, Korea
}

Background: The elderly have been reported to show anatomical and physiologic changes in the upper airway, which might affect the supraglottic airway (SGA) performance in geriatric populations. This study aimed to evaluate the clinical efficacy of the classic laryngeal mask airway (LMA-C) in the elderly compared with young adult patients.

Methods: Fifty patients aged 65-85 years (elderly group) and 50 patients aged 20-40 years (young group) who were scheduled for surgery using the LMA-C for general anesthesia were enrolled in this prospective, non-randomized, comparative study. Manipulations required during insertion, success rate, insertion time, oropharyngeal leak pressure, gastric insufflation, and intraoperative inadequate ventilation with the LMA-C were assessed. Fiberoptic evaluation was used to determine the position of the LMA-C.

Results: In the elderly group, the insertion success rate on the first attempt was significantly lower than that in the young group ( 84 vs. $96 \%, \mathrm{P}=0.02$ ). The insertion time in the elderly group was significantly longer than that in the young group ( $28.5 \pm 19.6$ vs. $22.2 \pm 6.4$ seconds, $\mathrm{P}=0.001)$. However, there was no difference in oropharyngeal leak pressure or fiberoptic grade between the two groups after proper placement of the LMA-C. During the surgery, inadequate ventilation events occurred more frequently in the elderly group than in the young group ( 31.3 vs. $4.0 \%, \mathrm{P}<0.001$ ).

Conclusions: The clinical efficacy of the LMA-C in elderly patients was inferior to that in young adult patients. Therefore, further studies are required to determine the type of SGA that can provide excellent clinical efficacy in the geriatric population.

Key Words: Aged, Laryngeal masks, Treatment outcome.

Corresponding author: Jae Hoon Lee, M.D., Ph.D.

Department of Anesthesiology and Pain Medicine, Severance Hospital, Anesthesia and Pain Research Institute, Yonsei University College of Medicine, 50, Yonsei-ro, Seodaemun-gu, Seoul 03722, Korea

Tel: 82-2-2228-2420, Fax: 82-2-312-7185

E-mail: neogens@yuhs.ac

ORCID: http://orcid.org/0000-0001-6679-2782

Received: June 5, 2015. Revised: July 10, 2015. Accepted: July 21, 2015.

Korean J Anesthesiol 2015 December 68(6): 568-574

http://dx.doi.org/10.4097/kjae.2015.68.6.568

(c) This is an open-access article distributed under the terms of the Creative Commons Attribution Non-Commercial License (http://creativecommons.org/ licenses/by-nc/4.0/), which permits unrestricted non-commercial use, distribution, and reproduction in any medium, provided the original work is properly cited. 


\section{Introduction}

Perioperative use of a supraglottic airway (SGA) is known to require a relatively low anesthetic depth, and results in less hemodynamic stimulation and a lower incidence of airway complications such as coughing and sore throat after emergence from anesthesia than endotracheal intubation [1]. Furthermore, an SGA could shorten the recovery time [1]. Due to these advantages, the SGA is useful, especially in geriatric patients, because elderly patients have declining functional reserve, suffer more from cardiopulmonary diseases, and are more sensitive to anesthetic drugs [2,3].

The cuff of the SGA is inserted through the mouth and placed between the tongue base, hypopharynx, and upper esophageal sphincter (UES) for proper ventilation [4]. Additionally, close contact between the cuff of the SGA and the adjacent tissues of the upper airway is essential for the ventilatory function of the SGA. Therefore, anatomical or physiological changes in the upper airway and UES may affect the performance of the SGA. Some previous studies reported structural and physiological changes in the pharyngeal airway and UES with increasing age [5-9]. In the elderly, the shape and size of the pharyngeal airway were found to be different from those in young adults [5-8]. There are changes in the function and structure of the UES with increasing age [9]. These changes with aging might affect the performance of the SGA in the elderly population.

However, despite abundant studies assessing the efficacy of the SGA in children according to the anatomic characteristics of the upper airway [10-12], to the best of our knowledge, very few studies have assessed the performance of the SGA in the geriatric population. Therefore, we designed this study to compare the clinical efficacy of the LMA Classic ${ }^{\mathrm{TM}}$ (LMA-C, Laryngeal Mask Company Ltd., Henley-on-Thames, UK) between elderly patients and young adult patients.

\section{Materials and Methods}

This prospective, non-randomized, comparative study was approved by the Institutional Ethics Committee and registered at ClinicalTrials.gov. Written informed consent was obtained from all subjects. Among the patients who were scheduled to undergo general anesthesia using the LMA-C for urologic and orthopedic surgeries expected to last less than 2 hours between December 2012 and November 2013, we enrolled 50 consecutive patients aged 20-40 years (young group). During the same period, 50 patients aged 65-85 years (elderly group) were enrolled consecutively. Exclusion criteria included American Society of Anesthesiologists physical status greater than III; known or predicted difficult airway (Mallampati class 4 , mouth opening $<3 \mathrm{~cm}$, or thyromental distance $<6 \mathrm{~cm}$ ); body mass index (BMI)
$>35 \mathrm{~kg} / \mathrm{m}^{2}$; increased risk of aspiration; gastro-esophageal reflux; obstructive sleep apnea syndrome; hyperreactive airway disease including asthma, chronic obstructive pulmonary disease, and recent upper respiratory disease; and neuromuscular disease.

All patients fasted for at least 8 hours. The size of the LMA$\mathrm{C}$ was determined based on the manufacturer's instructions and the patient's body weight. A size 3 LMA-C was used for patients weighing less than $50 \mathrm{~kg}$, a size 4 LMA-C was used for patients weighing $50-70 \mathrm{~kg}$, and a size 5 LMA-C was used for patients weighing more than $70 \mathrm{~kg}$. A standard pre-use test was performed. Then, the cuff of the LMA-C was partially inflated with half the amount of air recommended by the manufacturer (size 3 , $10 \mathrm{ml}$ of air; size 4, $15 \mathrm{ml}$ of air; and size 5, $20 \mathrm{ml}$ of air) [13-15]. All devices were lubricated with a water-soluble gel.

No premedication was administered to the patients. Upon arrival in the operating theater, the patient underwent monitoring including non-invasive blood pressure measurement, pulse oximetry, electrocardiography, and bispectral index (BIS). The patient's head was supported on a doughnut-shaped gel pad in a sniffing position. All patients were pre-oxygenated with $100 \%$ oxygen for $3 \mathrm{~min}$; then, anesthesia was induced with propofol $1.5 \mathrm{mg} / \mathrm{kg}$ and remifentanil $1.0 \mu \mathrm{g} / \mathrm{kg}$ for patients in the young group, and propofol $1.0 \mathrm{mg} / \mathrm{kg}$ and remifentanil $0.5 \mu \mathrm{g} / \mathrm{kg}$ for patients in the elderly group. Propofol $0.5 \mathrm{mg} / \mathrm{kg}$ was administered additionally until the patients lost consciousness. After loss of consciousness was confirmed by verbal stimuli, rocuronium $0.3 \mathrm{mg} / \mathrm{kg}$ was administered and the patients' lungs were manually ventilated through a facemask. Anesthesia was maintained with $1.0-4.0 \%$ of sevoflurane in an $\mathrm{O}_{2}$ /air mixture (fraction of inspired oxygen $=0.5$ ) to adjust the value of the BIS in the range of 40-60. LMA-C insertion was performed when the jaw was relaxed.

In all participants, the LMA-C was inserted and managed by three anesthesiologists who were experienced in using the LMA$\mathrm{C}$ and did not participate in this study. All data were assessed and recorded by an independent observer who did not know the objective of this study. At the first attempt, the LMA-C was inserted with the cuff partially inflated, using the midline approach [13-15]. If resistance was encountered during insertion, rightward or leftward rotation was performed during advancement [15-18]. Effective ventilation was confirmed by bilateral chest wall expansion and an appropriate capnographic trace after the cuff pressure of the LMA-C was adjusted to $60 \mathrm{cmH}_{2} \mathrm{O}$ using a cuff pressure gauge (VBM Medizintechnik GmbH, Sulz, Germany). If the attending anesthesiologist could not ventilate effectively through the LMA-C (inadequate ventilation: inability to generate more than $7-10 \mathrm{ml} / \mathrm{kg}$ tidal volume), manipulations to improve the position of the device, such as adjusting the depth of insertion (pushing or withdrawing the device slightly), thrusting the jaw, or extension/flexion of the head, were performed. 
If these manipulations were not effective, the anesthesiologist removed the LMA-C and tried to insert the LMA-C again at the second attempt. If effective ventilation was not obtained after reinsertion, the insertion attempt was considered a failure and the anesthesiologist managed the airway according to his or her own discretion. The insertion time was defined as the time from grasping the LMA-C to confirmation of effective ventilation.

After confirmation of effective ventilation, the lungs were mechanically ventilated with a tidal volume of $8 \mathrm{ml} / \mathrm{kg}$ at a rate of $8-15$ breaths/min. After $1 \mathrm{~min}$, mechanical ventilation was stopped and oropharyngeal leak pressure was measured by setting the expiratory valve of the breathing circle to $40 \mathrm{cmH}_{2} \mathrm{O}$ at a fresh gas flow of $3 \mathrm{~L} / \mathrm{min}$ and it was defined as the airway pressure at which equilibrium was reached and an audible leak at the mouth was ascertained $[19,20]$. At that time, the presence of gastric insufflation was also assessed by listening with a stethoscope over the epigastrium. Fiberoptic position of the LMA-C was assessed by passing a fiberscope through the LMA-C to a position just proximal to the mask aperture. The fiberoptic view was graded using the Brimacombe Score (1, vocal cords not visible; 2, vocal cords and anterior epiglottis visible; 3 , vocal cords and posterior epiglottis visible; 4 , vocal cords visible) [21].

When inadequate ventilation occurred during the surgical procedure, it was first managed by deepening the plane of anesthesia or through the administration of $0.1 \mathrm{mg} / \mathrm{kg}$ of rocuronium. In cases that could not be resolved by such a management, the previously mentioned manipulations to improve the position of the device were performed. After the end of the surgical procedure, the LMA-C was removed once the patient regained consciousness and responded to verbal commands. After removal, the LMA-C was inspected for any traces of blood. Throughout this investigation, desaturation was defined as $\mathrm{SpO}_{2}$ less than $95 \%$, and desaturation events were also recorded.

The independent observer assessed postoperative airway morbidity including sore throat, dysphonia, and dysphagia $24 \mathrm{~h}$ after surgery.

A previous study in adults excluding the elderly aged more than 65 years demonstrated that the mean \pm standard deviation (SD) oropharyngeal leak pressure of the LMA classic was $15.3 \pm 5.2 \mathrm{cmH}_{2} \mathrm{O}$ [22]. We considered that a difference of $20 \%$ in oropharyngeal leak pressure was clinically important, and to detect this difference with an alpha $=0.05$ and beta $=0.2$, at least 47 patients were required in each group. We decided to enrol 50 patients in each group, considering that some patients may drop out. Comparisons between the groups were performed with Student's t-test, the Mann-Whitney rank-sum test, $\chi^{2}$ test, and Fisher's exact test when appropriate. A P value $<0.05$ was considered statistically significant. All statistical analyses were performed using IBM SPSS Statistics version 20 (SPSS Inc., Chicago, IL, USA).

\section{Results}

Among the 55 young adult patients and 53 elderly patients who were screened for enrolment, 6 patients ( 5 young adult patients and 1 elderly patient) declined to participate in the study, and 2 elderly patients met the exclusion criteria of the study. Finally, 100 patients (50 patients in each group) participated in this study.

Table 1 shows the patient and airway-related characteristics. The patients' height, weight, and thyromental distance were greater in the young group than in the elderly group, and thus the composition of the LMA-C sizes inserted into the participants was different between the two groups. However, the BMI was comparable between the two groups. The number of patients with poor dentition was greater in the elderly group than in the young group. The duration of anesthesia and surgery was also longer in the young group than in the elderly group.

Parameters related to LMA-C insertion are shown in Table 2. Effective ventilation was achieved differently between the two groups ( $\mathrm{P}=0.002)$. Ventilation was obtained effectively at the first attempt without any manipulation in $42(84 \%)$ patients and $25(50 \%)$ patients and after a rotation maneuver in $6(12 \%)$ patients and 17 (34\%) patients in the young and elderly groups, respectively. The first attempt success rate was significantly lower in the elderly group ( 84 vs. $96 \%, \mathrm{P}=0.02$ ); thus, the insertion time was significantly greater in the elderly group than in the

Table 1. Patient and Airway-related Characteristics

\begin{tabular}{lccr}
\hline & $\begin{array}{c}\text { Young group } \\
(\mathrm{n}=50)\end{array}$ & $\begin{array}{c}\text { Elderly group } \\
(\mathrm{n}=50)\end{array}$ & P value \\
\hline Age $(\mathrm{yr})$ & $29.2 \pm 5.7$ & $71.2 \pm 5.0$ & $<0.001$ \\
Sex $(\mathrm{M} / \mathrm{F})$ & $42 / 8$ & $43 / 7$ & 1.000 \\
Height $(\mathrm{cm})$ & $173.3 \pm 6.9$ & $166.1 \pm 6.8$ & $<0.001$ \\
Weight $(\mathrm{kg})$ & $71.5 \pm 10.7$ & $65.5 \pm 10.4$ & 0.005 \\
Body mass index $\left(\mathrm{kg} / \mathrm{m}^{2}\right)$ & $23.9 \pm 3.0$ & $23.7 \pm 2.8$ & 0.731 \\
Current smoker & $20(40 \%)$ & $21(42 \%)$ & 1.000 \\
Mallampati class & & & 0.638 \\
$\quad 1$ & $37(74 \%)$ & $34(68 \%)$ & \\
2 & $10(20 \%)$ & $12(24 \%)$ & \\
$\quad 3$ & $3(6 \%)$ & $4(8 \%)$ & \\
Mouth opening $(\mathrm{cm})$ & $5.4 \pm 0.8$ & $5.2 \pm 0.8$ & 0.097 \\
Thyromental distance $(\mathrm{cm})$ & $7.9 \pm 0.8$ & $7.5 \pm 0.8$ & 0.044 \\
Poor dentition* & $0(0 \%)$ & $14(28 \%)$ & $<0.001$ \\
LMA-C size & & & $<0.001$ \\
3 & $2(4 \%)$ & $2(4 \%)$ & \\
4 & $15(30 \%)$ & $35(70 \%)$ & \\
$\quad 5$ & $33(66 \%)$ & $13(26 \%)$ & \\
Duration of anesthesia $(\mathrm{min})$ & $81.7 \pm 37.6$ & $64.8 \pm 30.7$ & 0.016 \\
Duration of surgery $(\mathrm{min})$ & $51.0 \pm 35.2$ & $33.2 \pm 28.5$ & 0.008 \\
\hline
\end{tabular}

Values are expressed as mean \pm SD or as number (\%). *Poor dentition includes participants with no teeth; missing, loose, or broken teeth; and partial or complete dentures. 
Table 2. Parameters Related to the LMA-C Insertion

\begin{tabular}{lccc}
\hline & $\begin{array}{c}\text { Young group } \\
(\mathrm{n}=50)\end{array}$ & $\begin{array}{c}\text { Elderly group } \\
(\mathrm{n}=50)\end{array}$ & P value \\
\hline First attempt success rate & $48(96 \%)$ & $42(84 \%)$ & 0.020 \\
Establishment of effective ventilation & & & 0.002 \\
On the first attempt without any manipulation & $42(84 \%)$ & $25(50 \%)$ \\
On the first attempt after a rotation maneuver & $6(12 \%)$ & $17(34 \%)$ \\
After adjusting the position or re-insertion & $2(4 \%)$ & $6(12 \%)$ & $2(4 \%)$ \\
Failure and endotracheal intubation & $0(0 \%)$ & $28.5 \pm 19.6^{*}$ & 0.001 \\
Insertion time (seconds) & $22.2 \pm 6.4$ & & \\
\hline
\end{tabular}

Values are expressed as number (\%) or as mean \pm SD. $* \mathrm{n}=48$.

Table 3. Performance of the LMA-C

\begin{tabular}{lccc}
\hline & $\begin{array}{c}\text { Young group } \\
(\mathrm{n}=50)\end{array}$ & $\begin{array}{c}\text { Elderly group } \\
(\mathrm{n}=48)\end{array}$ & P value \\
\hline Peak airway pressure $\left(\mathrm{cmH}_{2} \mathrm{O}\right)$ & $11.1 \pm 1.5$ & $11.7 \pm 2.0$ & 0.096 \\
Oropharyngeal leak pressure $\left(\mathrm{cmH}_{2} \mathrm{O}\right)$ & $18.7 \pm 4.3$ & $18.4 \pm 4.7$ & 0.705 \\
Gastric insufflation & $2(4.0 \%)$ & $3(6.2 \%)$ & 0.963 \\
Fiberoptic grade & & & 0.609 \\
1: vocal cords not visible & $14(28.0 \%)$ & $18(37.5 \%)$ & $7(14.6 \%)$ \\
2: vocal cords plus anterior epiglottis visible & $12(24.0 \%)$ & $9(18.8 \%)$ & \\
3: vocal cords plus posterior epiglottis visible & $9(18.0 \%)$ & $14(29.2 \%)$ & \\
4: only vocal cords visible & $15(30.0 \%)$ & & \\
\hline
\end{tabular}

Values are expressed as mean \pm SD or as number (\%).

Table 4. Blood-tinged LMA-C after Removal and Postoperative Airway Morbidity

\begin{tabular}{lccc}
\hline & $\begin{array}{c}\text { Young group } \\
(\mathrm{n}=50)\end{array}$ & $\begin{array}{c}\text { Elderly group } \\
(\mathrm{n}=48)\end{array}$ & P value \\
\hline $\begin{array}{l}\text { Blood-tinged LMA-C after removal } \\
\text { Morbidity at 24 h after surgery }\end{array}$ & $6(12.0 \%)$ & $1(2.1 \%)$ & 0.112 \\
$\quad$ Sore throat & $6(12.0 \%)$ & $5(10.4 \%)$ & 0.804 \\
Dysphonia & $0(0 \%)$ & $2(4.2 \%)$ & 0.237 \\
Dysphagia & $1(2.0 \%)$ & $0(0 \%)$ & 1.000 \\
\hline
\end{tabular}

Values are expressed as number (\%).

young group. Moreover, in 2 patients from the elderly group, proper placement of the LMA-C failed despite a second attempt; finally, endotracheal intubation was performed. They were excluded from further assessments. However, there was no case of desaturation during anesthetic induction.

Table 3 demonstrates the performance of the LMA-C after proper placement for effective ventilation. The oropharyngeal leak pressure and fiberoptic grade were comparable between the two groups.

During the surgery, inadequate ventilation events requiring adjustment of the LMA-C position occurred in 15 (31.3\%) patients from the elderly group and in $2(4.0 \%)$ patients from the young group $(\mathrm{P}<0.001)$. During anesthetic emergence, there was 1 case of desaturation in the elderly group, probably caused by a laryngeal spasm lasting less than $1 \mathrm{~min}$. However, the patient recovered without any significant morbidity.

Table 4 describes the number of patients with a blood-tinged LMA-C after removal and postoperative airway morbidity, which were not significantly different between the two groups.

\section{Discussion}

This study demonstrated that the success rate of adequate ventilation on the first attempt when placing an LMA-C was significantly lower in elderly patients than in young adult patients. Moreover, the elderly patients required more rotatory manipulations and position adjustments for insertion and placement of the LMA-C for adequate ventilation compared to the young 
patients. Therefore, it took more time to insert and place the LMA-C in the proper position in elderly patients than in young patients. Additionally, episodes of inadequate ventilation requiring LMA-C position adjustment during surgery occurred more frequently in the elderly patients than in the young patients.

Our findings with respect to the first attempt success rate of LMA-C insertion and adequate ventilation in the young group (96\%) compares well with the results of previous studies [15,23]. Previous studies demonstrated that the first attempt success rate in adults (mean age: 39.7-45.1 years) was 96.7-98.0\% [15,23]. In our study, the number of elderly patients who needed further adjustment of the LMA-C position after initial insertion was greater than that of young patients. Therefore, the first attempt success rate among the elderly patients was $84 \%$ and it was significantly lower than that among the young patients. According to a magnetic resonance imaging study, the cuff of the SGA should be located vertically between the tongue base and the UES and horizontally between both lateral pharyngeal walls [4]. Previous studies reported structural changes in the pharyngeal airway with increasing age. The volume or cross-sectional area of the pharyngeal airway may be greater in the elderly than in the young [5-7]. The length of the pharyngeal airway was shown to be longer in the elderly $[7,8]$. These changes may increase or transform the potential space in which the cuff of the LMA-C is placed. Moreover, the resting pressure of the UES was lower and the length of the UES high-pressure zone was shorter in the elderly than in the young [9], which may affect the depth of insertion of the tip of the LMA-C cuff into the UES. Therefore, changes related to aging may be associated with difficulty in obtaining proper positioning for adequate ventilation on the first attempt in elderly patients. However, when the LMA-C was placed in the proper position for adequate ventilation, the ventilation performance of the LMA-C was comparable between the elderly and young patients; there was no difference in the oropharyngeal leak pressure, the incidence of gastric insufflation, and the fiberoptically determined cuff position.

In this study, the LMA-C was inserted with a partially inflated cuff. Although it was first reported that the LMA-C should be inserted with the cuff fully deflated, previous studies demonstrated that an LMA-C with the cuff partially inflated could be more successfully and easily placed [13-15]. Additionally, studies comparing the LMA-C with another type of SGA demonstrated that fully deflated LMA-Cs had lower first attempt success rates $[22,24]$. Furthermore, even in pediatric patients who have their own upper airway characteristics, insertion of the LMA-C with the cuff partially inflated was associated with a shorter insertion time and higher success rate $[25,26]$. Therefore, we adopted this technique. However, our study showed that a greater number of elderly patients required rotatory manipulation during LMA$\mathrm{C}$ advancement than young patients, which means that there was resistance during LMA-C insertion in a greater number of patients among the elderly group than among the young group, although the elderly were shown to have greater pharyngeal airway space [5-8]. This finding could not be explained by the anatomical and physiological changes in the upper airway with aging. However, this finding might imply that insertion of the LMA-C with the cuff partially inflated is less useful in elderly patients because the technique increases the size of the LMA-C cuff and thus may increase resistance during the advancement of the LMA-C. Of note, Ezri et al. [27] reported a higher rate (87.5\%) of easy insertion (without any manipulations) with a fully deflated LMA-C cuff in elderly patients compared to our study (50\%, success on the first attempt without manipulations). However, we cannot conclude that an insertion technique with a fully deflated cuff is appropriate in the elderly because the previous study did not describe the definite method of assessment and enrolled relatively younger patients in the elderly group (mean age: 66 years) compared to our study (the average age of the elderly patients in the present study was 71 years).

Our study reported intraoperative events of inadequate ventilation requiring LMA-C position adjustment. Because these events were not resolved by deepening the anesthesia or through the administration of a muscle relaxant, displacement of the LMA-C cuff was the likely cause of inadequate ventilation. In the elderly group, the number of patients with inadequate ventilation resulting from displacement during surgery was significantly greater than that in the young group, although the duration of surgery was shorter in the elderly group than in the young group. The previously mentioned changes in the pharyngeal airway with aging might be associated with higher occurrence of inadequate ventilation in the elderly group. Furthermore, poor dental status in the elderly group may need more attention in order to maintain the position of the LMA-C and thus it might cause inadequate ventilation events.

Performing rotatory manipulations and position adjustments after the first LMA-C insertion attempt can increase the time required to obtain proper ventilation. As a result, the insertion time for adequate ventilation was significantly greater in elderly patients than in young patients in this study. Additionally, inadequate ventilation during the surgical procedure occurred more frequently in the elderly than in the young. These situations may increase the chance of hypoxic events in all elderly patients and can be dangerous, especially in multi-morbid elderly patients. Moreover, this study demonstrated that the clinical efficacy of the LMA-C in the elderly may be different from that in young patients, and the elderly have their own airway characteristics. Therefore, the performances of different types of SGAs need to be evaluated separately in geriatric populations.

This study has some limitations. The clinical efficacy of the LMA-C was compared between elderly and young adult pa- 
tients, and middle-aged patients (aged 41-64 years) were not enrolled in this study. Because of the substantial generation gap between the two groups, there was a significant difference in physical constitution between the two groups, and thus the size of the LMA-C used in this study was not comparable between the elderly and the young. The application of a larger-sized LMA-C in the young patients might have affected the results of this study. However, in this study, the size of the LMA-C was determined based on the manufacturer's recommendations. Also, the rate of poor dental status was higher in the elderly group. Poor dentition may have affected the performance of the LMA$\mathrm{C}$ in elderly patients. However, the first attempt success rate of the LMA-C was $88.8 \%$ and the rate of intraoperative inadequate ventilation was $33.3 \%$ in 36 elderly patients with good dentition. Thus, the performance of the LMA-C in elderly patients with good dentition was inadequate compared to that in the young patients.

In conclusion, the LMA-C is less effective in elderly patients than in young adult patients. Therefore, further studies that compare and assess the clinical efficacy of other types of SGAs are needed in order to aid proper selection of an SGA in the elderly.

\section{ORCID}

Eun Mi Kim, http://orcid.org/0000-0003-0724-7607

Jae Hoon Lee, http://orcid.org/0000-0001-6679-2782

\section{References}

1. Yu SH, Beirne OR. Laryngeal mask airways have a lower risk of airway complications compared with endotracheal intubation: a systematic review. J Oral Maxillofac Surg 2010; 68: 2359-76.

2. Cook DJ, Rooke GA. Priorities in perioperative geriatrics. Anesth Analg 2003; 96: 1823-36.

3. Jin F, Chung F. Minimizing perioperative adverse events in the elderly. Br J Anaesth 2001; 87: 608-24.

4. Russo SG, Cremer S, Eich C, Jipp M, Cohnen J, Strack M, et al. Magnetic resonance imaging study of the in vivo position of the extraglottic airway devices i-gel and LMA-Supreme in anaesthetized human volunteers. Br J Anaesth 2012; 109: 996-1004.

5. Burger CD, Stanson AW, Sheedy PF 2nd, Daniels BK, Shepard JW Jr. Fast-computed tomography evaluation of age-related changes in upper airway structure and function in normal men. Am Rev Respir Dis 1992; 145: 846-52.

6. Mayer P, Pepin JL, Bettega G, Veale D, Ferretti G, Deschaux C, et al. Relationship between body mass index, age and upper airway measurements in snorers and sleep apnoea patients. Eur Respir J 1996; 9: 1801-9.

7. Malhotra A, Huang Y, Fogel R, Lazic S, Pillar G, Jakab M, et al. Aging influences on pharyngeal anatomy and physiology: the predisposition to pharyngeal collapse. Am J Med 2006; 119: 72.e9-14.

8. Shigeta Y, Ogawa T, Venturin J, Nguyen M, Clark GT, Enciso R. Gender- and age-based differences in computerized tomographic measurements of the orophaynx. Oral Surg Oral Med Oral Pathol Oral Radiol Endod 2008; 106: 563-70.

9. Bardan E, Xie P, Brasseur J, Dua K, Ulualp SO, Kern M, et al. Effect of ageing on the upper and lower oesophageal sphincters. Eur J Gastroenterol Hepatol 2000; 12: 1221-5.

10. Kim MS, Oh JT, Min JY, Lee KH, Lee JR. A randomised comparison of the i- gel ${ }^{\mathrm{TM}}$ and the Laryngeal Mask Airway Classic ${ }^{\mathrm{TM}}$ in infants. Anaesthesia 2014; 69: 362-7.

11. Lee JR, Kim MS, Kim JT, Byon HJ, Park YH, Kim HS, et al. A randomised trial comparing the i-gel (TM) with the LMA Classic (TM) in children. Anaesthesia 2012; 67: 606-11.

12. Shimbori H, Ono K, Miwa T, Morimura N, Noguchi M, Hiroki K. Comparison of the LMA-ProSeal and LMA-Classic in children. Br J Anaesth 2004; 93: 528-31.

13. Matta BF, Marsh DS, Nevin M. Laryngeal mask airway: a more successful method of insertion. J Clin Anesth 1995; 7: 132-5.

14. Kim MS, Lee JR, Shin YS, Chung JW, Lee KH, Ahn KR. Comparison of 2 cuff inflation methods of laryngeal mask airway Classic for safe use without cuff manometer in adults. Am J Emerg Med 2014; 32: 237-42.

15. Lin BC, Wu RS, Chen KB, Yang MH, Lo YC, Chiang YY. A comparison of the classic and a modified laryngeal mask airway (OPLAC $\left.{ }^{\mathrm{TM}}\right)$ in adult patients. Anesth Analg 2011; 112: 539-44.

16. Dingley J, Asai T. Insertion methods of the laryngeal mask airway. A survey of current practice in Wales. Anaesthesia 1996; 51: 596-9.

17. Matthew PJ, Bala I. Comparison of lateral and standard techniques of laryngeal mask airway insertion in adults. Anaesth Intensive Care 2008; 36: 914-5.

18. Kundra P, Deepak R, Ravishankar M. Laryngeal mask insertion in children: a rational approach. Paediatr Anaesth 2003; $13: 685-90$.

19. Francksen H, Renner J, Hanss R, Scholz J, Doerges V, Bein B. A comparison of the i-gel with the LMA-Unique in non-paralysed anaesthetised adult patients. Anaesthesia 2009; 64: 1118-24.

20. Keller C, Brimacombe JR, Keller K, Morris R. Comparison of four methods for assessing airway sealing pressure with the laryngeal mask airway in adult patients. Br J Anaesth 1999; 82: 286-7.

21. Brimacombe J, Berry A. A proposed fiber-optic scoring system to standardize the assessment of laryngeal mask airway position. Anesth Analg 1993; 76: 457.

22. Shariffuddin II, Wang CY. Randomised crossover comparison of the Ambu AuraOnce Laryngeal Mask with the LMA Classic laryngeal 
mask airway in paralysed anaesthetised patients. Anaesthesia 2008; 63: 82-5.

23. Alexiev V, Ochana A, Abdelrahman D, Coyne J, McDonnell JG, O’Toole DP, et al. Comparison of the Baska $\left({ }^{\circledR}\right)$ mask with the single-use laryngeal mask airway in low-risk female patients undergoing ambulatory surgery. Anaesthesia 2013; 68: 1026-32.

24. Janakiraman C, Chethan DB, Wilkes AR, Stacey MR, Goodwin N. A randomised crossover trial comparing the i-gel supraglottic airway and classic laryngeal mask airway. Anaesthesia 2009; 64: 674-8.

25. O’Neill B, Templeton JJ, Caramico L, Schreiner MS. The laryngeal mask airway in pediatric patients: factors affecting ease of use during insertion and emergence. Anesth Analg 1994; 78: 659-62.

26. Ghai B, Wig J. Comparison of different techniques of laryngeal mask placement in children. Curr Opin Anaesthesiol 2009; 22: 400-4.

27. Ezri T, Ady N, Szmuk P, Glanz L, Shklar B, Katz J, et al. Use of cuffed oropharyngeal vs laryngeal mask airway in elderly patients. Can J Anaesth 1999; 46: 363-7. 